\title{
Multi-wavelength photoacoustic imaging for monitoring lesion formation during high-intensity focused ultrasound therapy
}

\author{
Xun $\mathrm{Wu}^{* \mathrm{a}}$, Jean Sanders ${ }^{\mathrm{a}}$, Murat Dundar ${ }^{\mathrm{b}}$, Ömer Oralkan ${ }^{\mathrm{a}}$, \\ ${ }^{a}$ Department of Electrical and Computer Engineering, North Carolina State University, Raleigh, NC \\ 27606, USA; ${ }^{b}$ Department of Computer and Information Science, Indiana University-Purdue \\ University Indianapolis, Indianapolis, IN 46202, USA
}

\begin{abstract}
Photoacoustic imaging (PAI) can be used to monitor lesion formation during high-intensity focused ultrasound (HIFU) therapy because HIFU changes the optical absorption spectrum (OAS) of the tissue. However, in traditional PAI, the change could be too subtle to be observed either because the OAS does not change very significantly at the imaging wavelength or due to low signal-to-noise ratio in general. We propose a machine-learning-based method for lesion monitoring with multi-wavelength PAI (MWPAI), where PAI is repeated at a sequence of wavelengths and a stack of multi-wavelength photoacoustic (MWPA) images is acquired. Each pixel is represented by a vector and each element in the vector reflects the optical absorption at the corresponding wavelength. Based on the MWPA images, a classifier is trained to classify pixels into two categories: ablated and non-ablated. In our experiment, we create a lesion on a block of bovine tissue with a HIFU transducer, followed by MWPAI in the $690 \mathrm{~nm}$ to $950 \mathrm{~nm}$ wavelength range, with a step size of $5 \mathrm{~nm}$. In the MWPA images, some of the ablated and non-ablated pixels are cropped and fed to a neural network (NN) as training examples. The NN is then applied to several groups of MWPA images and the results show that the lesions can be identified clearly. To apply MWPAI in/near real-time, sequential feature selection is performed and the number of wavelengths is decreased from 53 to 5 while retaining adequate performance. With a fast-switching tunable laser, the method can be implemented in/near real-time.
\end{abstract}

Keywords: Multi-wavelength photoacoustic imaging, high-intensity focused ultrasound therapy, lesion formation monitoring, machine learning

\section{INTRODUCTION}

High-intensity focused ultrasound (HIFU) therapy, or focused ultrasound surgery (FUS), is an emerging medical procedure that heats and destroys diseased tissue noninvasively by focusing acoustic energy underneath a patient's skin. Reliable and accurate therapy monitoring is indispensable for safe and effective HIFU therapy. Ultrasound imaging has been used to assist HIFU therapy (USgFUS) because the lesions created by HIFU show up in ultrasound images as hyperechoic regions ${ }^{1}$. Besides, ultrasound imaging is advantageous for being portable, real-time, and low-cost. However, ultrasound-based monitoring is not widely-used due to its lack of precision. Magnetic resonance imaging (MRI) provides a more precise approach (MRgFUS) by monitoring temperature distribution during HIFU therapy ${ }^{2}$, but MRI is bulky, slow, and expensive ${ }^{3}$.

Photoacoustic imaging (PAI) combines the contrast of optical absorption with the deep penetration and high spatial resolution of ultrasound imaging ${ }^{4}$. PAI is a good alternative for lesion monitoring during ablations because HIFU therapy changes the optical absorption spectrum (OAS) of tissue ${ }^{5}$, which can be detected with PAI. However, the change could be too subtle to be observed either because the OAS does not change very significantly at a particular imaging wavelength or due to low signal-to-noise ratio in general. Photoacoustic spectroscopy measures optical property at more than one wavelength ${ }^{6}$ and can potentially make the OAS change easier to be detected in PAI. A previous study showed visualization of HIFU lesions with multi-wavelength PAI (MWPAI) using a correlation-based algorithm ${ }^{7}$. In this study, we develop a machine-learning-based approach to process multi-wavelength photoacoustic (MWPA) images and identify lesion formation in tissue. The following section presents a theoretical background for the developed approach. Experimental methods are described next, followed by the presentation of the results and related discussion.

*xwu12@ncsu.edu; phone1919607-5823

Photons Plus Ultrasound: Imaging and Sensing 2017, edited by Alexander A. Oraevsky, Lihong V. Wang, Proc. of SPIE Vol. 10064, 100644A · @ 2017 SPIE · CCC code: 1605-7422/17/\$18 · doi: 10.1117/12.2248739 


\section{THEORETICAL BACKGROUND}

\subsection{Photoacoustic effect}

The physical basis for PAI, photoacoustic (PA) effect, refers to the generation of acoustic waves caused by the absorption of electromagnetic energy. A short laser pulse causes thermal expansion of tissue, which induces a transient pressure rise $P$. It can be calculated as ${ }^{7,8}$ :

$$
P(\vec{r}, T, \lambda)=\left(\frac{\beta(T) v_{s}^{2}(T)}{C_{p}(T)}\right) \mu_{a b s}(\lambda) F_{0}(\lambda) \eta(\vec{r})
$$

where $\beta(T)$ is the volume expansion coefficient, $v_{s}(T)$ is the speed of sound, $C_{p}(T)$ is the isobaric heat capacity, $\mu_{a b s}(\lambda)$ is the optical absorption coefficient, $F_{0}(\lambda)$ is the incident laser fluence, $\eta(\vec{r})$ is the percentage of laser fluence that has reached position $\vec{r} \cdot \beta(T) \nu_{s}^{2}(T) / C_{p}(T)$ is referred to as the Grüneisen coefficient $\Gamma(T)$, which is a function of temperature $T$. So, equation (1) can be written as:

$$
P(\vec{r}, T, \lambda)=\Gamma(T) \mu_{a b s}(\lambda) F_{0}(\lambda) \eta(\vec{r})
$$

\subsection{Photoacoustic imaging system}

The pixel values in a PA image linearly scale with the pressure rise caused by PA effect due to the linearity of the PAI system ${ }^{9}$. So, the pixel value at $\vec{r}$ is proportional to the pressure rise at the corresponding location in the imaging field:

$$
v(\vec{r})=E(\vec{r}) P(\vec{r})+N
$$

$E(\vec{r})$ is a constant that depends on the PAI system and the location $\vec{r} . N$ is the system's added noise. Combining (2) and (3):

$$
v(\vec{r}, T, \lambda)=E(\vec{r}) \Gamma(T) \mu_{a b s}(\lambda) F_{0}(\lambda) \eta(\vec{r})+N
$$

\subsection{Multi-wavelength photoacoustic imaging and optical absorption spectrum}

In MWPAI, PAI is repeated at a sequence of wavelength $\lambda_{i}(i=1,2, \ldots, N)$. Each pixel is represented by an $\mathrm{N}$-element vector where element $e$ is the pixel value at wavelength $\lambda_{i}$ :

$$
e\left(\vec{r}, T, \lambda_{i}\right)=E(\vec{r}) \Gamma(T) \mu_{a b s}\left(\lambda_{i}\right) F_{0}\left(\lambda_{i}\right) \eta(\vec{r})+N
$$

If temperature $T$ is stable and system noise $N$ is low enough to be negligible:

$$
e\left(\lambda_{i}\right)=C(\vec{r}) \mu_{a b s}\left(\lambda_{i}\right) F_{0}\left(\lambda_{i}\right)
$$

where $C(\vec{r})=E(\vec{r}) \Gamma(T) \eta(\vec{r})$. If the laser fluence $F_{0}\left(\lambda_{i}\right)$ at each wavelength is known and used to normalize each element in the vector. The normalized element is:

$$
e_{\text {norm }}\left(\lambda_{i}\right)=C(\vec{r}) \mu_{\text {abs }}\left(\lambda_{i}\right)
$$

For a specific type of tissue, we assume $\mu_{a b s}(\lambda)$ reaches its peak value at $\lambda_{\max }$. By normalizing the element with $e_{\text {norm }}\left(\lambda_{\max }\right)$, the normalized element value can be written as:

$$
e_{\text {norm }}\left(\lambda_{i}\right)=\frac{\mu_{a b s}\left(\lambda_{i}\right)}{\mu_{a b s}\left(\lambda_{\max }\right)}
$$


From equation (8), we can see that the pixel value, or vector, at a specific location in MWPAI can be converted to its optical absorption spectrum that is normalized to its peak value, regardless of its location.

Neural networks (NNs) provide a powerful computational tool for classification. One study shows the ability of NNs to assist ovarian cancer diagnosis by classifying co-registered ultrasound and photoacoustic images ${ }^{10}$. In this study, we use the $\mathrm{NN}$ approach as our fundamental tool for pixel classification.

\section{METHODS}

\subsection{Experimental setup}

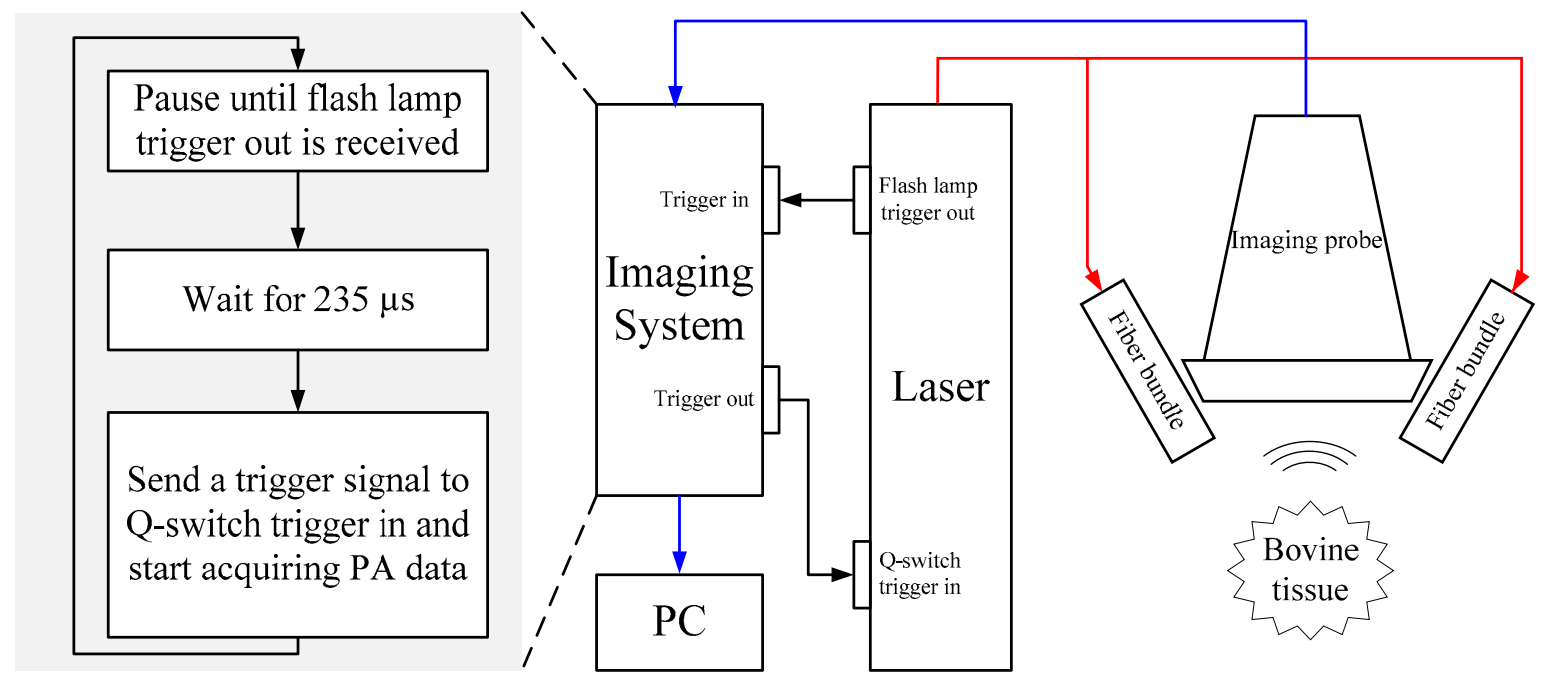

Figure 1. Experimental setup.

Figure 1 shows the block diagram of the experimental setup and the triggering scheme. A programmable imaging system (Model Vantage 64 LE, Verasonics Inc., Kirkland, WA), a PC (Model Precision T7910, Dell Inc., Round Rock, TX), and a linear ultrasound transducer array (Model L7-4, Philips, Bothell, WA) are used for PA data acquisition. The imaging system has 64 parallel receive channels with 2:1 multiplexing capability to receive from all of the 128 elements of the linear array. Therefore, the laser has to fire twice to acquire the data to reconstruct a complete frame at a certain wavelength. A programmable laser system (Model Phocus Mobile, Opotek Inc., Carlsbad, CA) with a customized dualoutput fiber is used for laser illumination. The laser system is programmed to scan from wavelength $690 \mathrm{~nm}$ to $950 \mathrm{~nm}$ with a step size of $5 \mathrm{~nm}$ and fire twice at each wavelength. The width of the laser pulse is $5 \mathrm{~ns}$ and the repetition rate is $20 \mathrm{~Hz}$. The energy per pulse at the output of the dual-output fiber is different at different wavelengths. The minimum is $7.93 \mathrm{~mJ}$ at $950 \mathrm{~nm}$ and the maximum is $14.74 \mathrm{~mJ}$ at $715 \mathrm{~nm}$. The dual-output fiber has an exit aperture of $40 \mathrm{~mm}$ by 0.25 $\mathrm{mm}$ in each branch. The flash lamp trigger output signal of the laser serves as the master clock that drives the system.
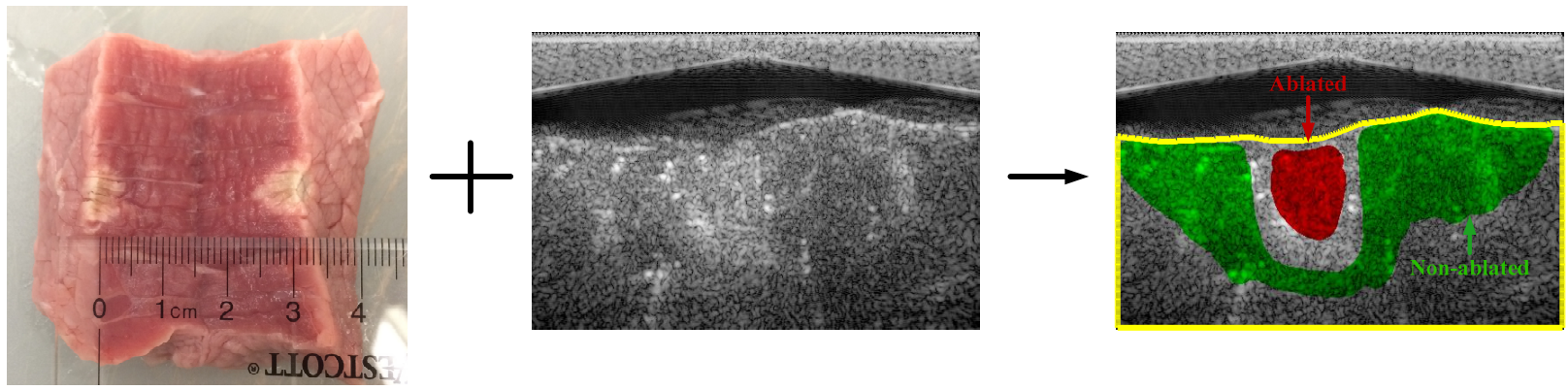

Figure 2. PA image preprocessing. 
Table 1. Performance of the classifier with all wavelengths.

\begin{tabular}{|c|c|c|c|c|c|}
\hline Dataset & Sensitivity (\%) & Specificity (\%) & PPV (\%) & NPV (\%) & Accuracy (\%) \\
\hline Group 1 & 75.2748 & 73.5378 & 73.9896 & 74.8377 & 74.4063 \\
\hline Group 2 & 75.9613 & 71.9018 & 72.9979 & 74.9442 & 73.9316 \\
\hline Group 3 & 76.8025 & 69.7957 & 71.7735 & 75.0546 & 73.2991 \\
\hline
\end{tabular}

Table 2. Performance of the classifier with reduced wavelengths.

\begin{tabular}{|c|c|c|c|c|c|}
\hline Dataset & Sensitivity (\%) & Specificity (\%) & PPV (\%) & NPV (\%) & Accuracy (\%) \\
\hline Group 1 & 65.2734 & 62.4644 & 63.4900 & 64.2698 & 63.8689 \\
\hline Group 2 & 69.5246 & 63.4744 & 65.5582 & 67.5620 & 66.4995 \\
\hline Group 3 & 67.6829 & 65.3100 & 66.1141 & 66.8974 & 66.4965 \\
\hline
\end{tabular}

Table 3. Performance of the classifier with reduced wavelengths and combined pixels.

\begin{tabular}{|c|c|c|c|c|c|}
\hline Dataset & Sensitivity (\%) & Specificity (\%) & PPV (\%) & NPV (\%) & Accuracy (\%) \\
\hline Group 1 & 70.1859 & 69.6838 & 69.8353 & 70.0355 & 69.9349 \\
\hline Group 2 & 72.5026 & 70.4911 & 71.0730 & 71.9381 & 71.4969 \\
\hline Group 3 & 68.9472 & 71.8497 & 71.0082 & 69.8231 & 70.3985 \\
\hline
\end{tabular}

Table 4. Performance of the classifier with full wavelengths and combined pixels.

\begin{tabular}{|c|c|c|c|c|c|}
\hline Dataset & Sensitivity (\%) & Specificity (\%) & PPV (\%) & NPV (\%) & Accuracy (\%) \\
\hline Group 1 & 93.9748 & 83.6341 & 85.1679 & 93.2799 & 88.8045 \\
\hline Group 2 & 74.2738 & 80.1097 & 78.8770 & 75.6924 & 77.1917 \\
\hline Group 3 & 69.6913 & 79.4411 & 77.2201 & 72.3839 & 74.5662 \\
\hline
\end{tabular}

\subsection{Experimental procedure and data acquisition}

Sample ex-vivo bovine tissue is cut into blocks (approximately $4.0 \mathrm{~cm} \times 4.0 \mathrm{~cm} \times 3.0 \mathrm{~cm}$ ). A HIFU transducer $(5-\mathrm{MHz}$, 19-mm circular aperture, 15-mm focal distance, Precision Acoustics Ltd., Dorchester, Dorset, UK) is used to create a visible lesion on the block before it is placed in room-temperature water to be imaged.

Every iteration of PA data acquisition is initiated by the rising edge of the flash lamp trigger signal from the laser. After receiving the trigger signal, the imaging system waits for $235 \mu \mathrm{s}$, sends a trigger signal to the Q-switch trigger-in port of the laser so that it fires, and starts acquiring PA data concurrently.

A hundred frames of photoacoustic data are acquired at each wavelength to reconstruct MWPA images. One frame of ultrasound data is also acquired to reconstruct a co-registered ultrasound image that can be used for anatomical structure matching.

\subsection{Data processing}

Standard delay-and-sum beamforming is applied to both ultrasound and photoacoustic data for image reconstruction. Photoacoustic frames of the same wavelength are averaged before image reconstruction for higher signal-to-noise ratio. Based on the ultrasound image, the tissue part of the image is cropped as the area where pixels are to be classified. 
Besides, some pixels of ablated and non-ablated tissue are selected for training and testing. The ground truth for training examples is generated by visually co-registering the ultrasound image and the photograph of the lesion after the tissue is cut open and manually delineating it. Figure 2 shows the cropped region as well as the marked pixels. The pixels in the yellow frame are cropped tissue pixels. The red and green regions are ablated and non-ablated tissue pixels, respectively, that are manually marked. They are used to train the NN.

The laser pulse energy is measured beforehand and used to normalize each vector to eliminate the effect of variation of incident laser fluence due to wavelength. Then each vector is normalized to its maximum element. The training examples are fed to a 3-layer NN. The number of neurons in each layer is 53,159, and 1. After the training is completed, the $\mathrm{NN}$ is tested to check the classification performance. Three groups of datasets are used for testing. Group 1 is the MWPA image where training examples are selected. Group 2 contains three MWPA images of the same block of bovine tissue as group 1 but at different imaging planes. Group 3 contains eight MWPA images of two other blocks of bovine tissue. After the classification is finished, the density of "ablated" pixels, which is the number of "ablated" pixels over the number of all the pixels in the region of interest, inside the $21 \times 21$ window that centers each pixel is calculated and thus a density map is generated. Then the 0.75 -density contour is drawn on the map to delineate lesion areas.
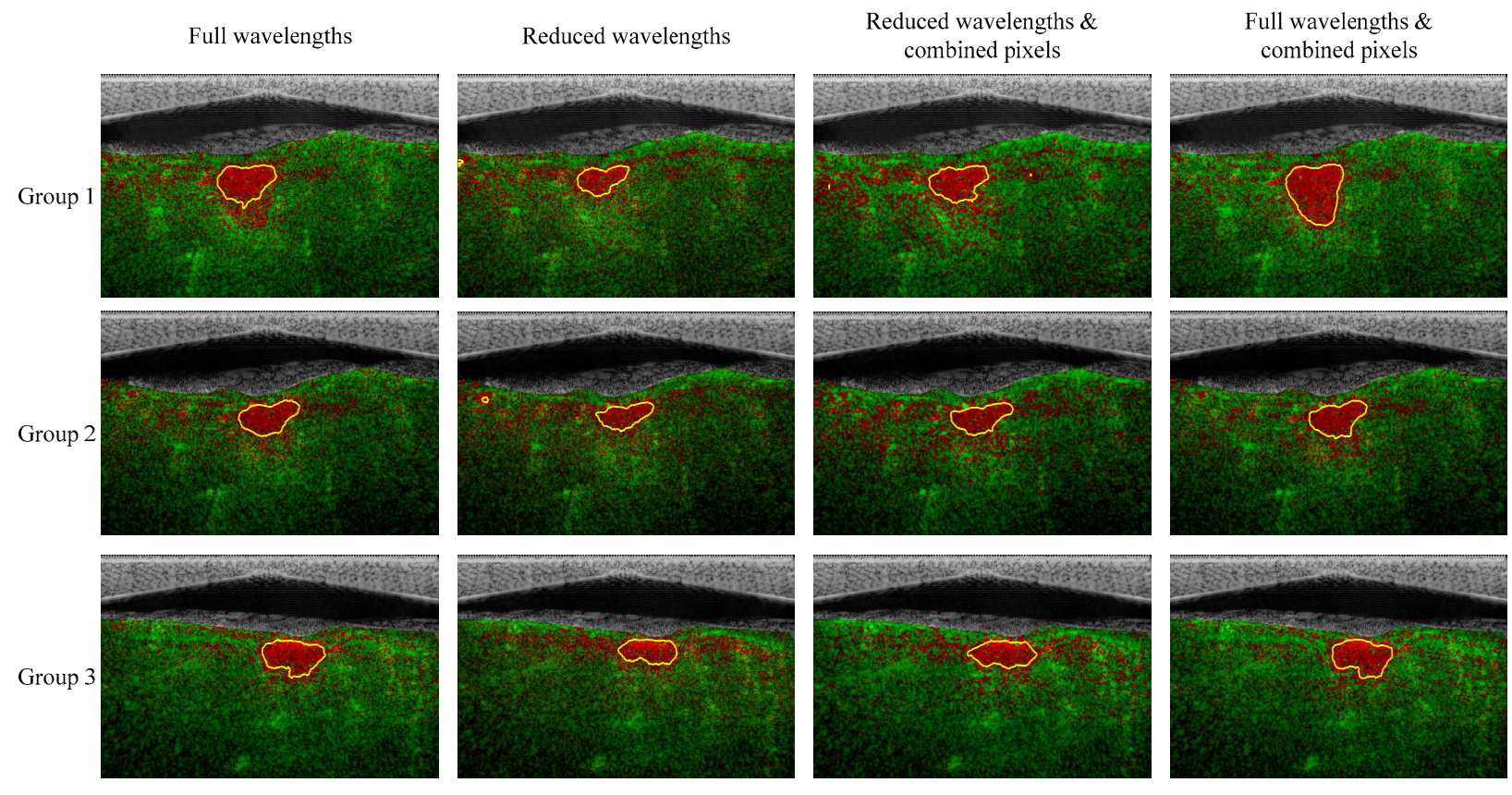

Figure 3. Classification result.

\subsection{Wavelength selection}

In order to facilitate real-time implementation of this machine-learning-based lesion identification approach, reducing the number of wavelengths is desired because it decreases the number of frames required for each MWPA image and increases the frame rate. In this study, we choose a sequential feature selection algorithm ${ }^{11}$. It keeps adding new wavelengths until the number of misclassifications stops decreasing. The algorithm gives 10 out of 53 wavelengths that are the most relevant to classification, and we choose the first 5 wavelengths as selected features.

\subsection{Improvement in classification by taking into account texture and spatial information}

Fewer wavelengths facilitate real-time implementation, but tend to degrade classification performance at the same time. In order to boost the performance when using fewer wavelengths, the pixels in a $3 \times 3$ window that centers each pixel are combined to represent it before feeding it to the NN. With this approach, texture and spatial information is also taken into consideration. 


\section{RESULT AND DISCUSSION}

Figure 3 shows the classification results for different groups of datasets. Tables 1,2, 3, and 4 show the sensitivity, specificity, positive predictive value (PPV), negative predictive value (NPV), and accuracy for each dataset with different classifier configuration. As we can see, the basic classifier is able to identify pixels of ablated tissue. After sequential selection, $690 \mathrm{~nm}, 780 \mathrm{~nm}, 785 \mathrm{~nm}, 845 \mathrm{~nm}$, and $865 \mathrm{~nm}$ are selected as the 5 most relevant wavelengths to classification. There is some performance degradation when using fewer wavelengths, but it can be compensated to some extent by using combined PA image pixels. The best performance is achieved when using combined PA image pixels at all wavelengths.

\section{DISCUSSION}

The constraints for classification performance includes the following. First, the imaging system noise could degrade the performance. The second constraint is the penetration depth of PAI. It could be increased by boosting the laser pulse energy. In this study, we assume that each pixel is either ablated or non-ablated while it could actually be somewhere in between. Probabilistic classification could be applied to solve this issue. In our study, the ground truth for training examples is generated by visually co-registering the ultrasound image and the photograph of the lesion after the tissue is cut open and manually delineating it. This method could be not accurate enough.

There is also room for improvement by using a more sophisticated classifier. Other classification methods such as support vector machine could also be used to achieve better performance than $\mathrm{NN}^{10}$.

\section{CONCLUSION}

This study develops a machine-learning-based approach for lesion identification during HIFU therapy. With this approach, lesions in ex-vivo bovine tissue can be identified clearly. Sequential feature selection decreases the number of wavelengths and facilitates real-time implementation. With our programmable laser system and a high-end computing platform, the approach can be implemented in real-time.

\section{ACKNOWLEDGMENT}

This work is supported by the National Institutes of Health under grant 1R01HL11740.

\section{REFERENCES}

[1] S. Vaezy, X. Shi, R. W. Martin, E. Chi, P. I. Nelson, M. R. Bailey, and L. A. Crum, "Real-time visualization of high-intensity focused ultrasound treatment using ultrasound imaging," Ultrasound in Medicine \& Biology, vol. 27, no. 1, pp. 33-42, Jan. 2011.

[2] C. Mougenot, R. Salomir, J. Palussière, N. Grenier, and C. T. W. Moonen, "Automatic spatial and temporal temperature control for MR-guided focused ultrasound using fast 3D MR thermometry and multispiral trajectory of the focal point," Magnetic Resonance in Medicine, vol. 52, no. 5, pp. 1005-1015, Nov. 2004.

[3] P. Steiner, R. Botnar, B. Dubno, G. G. Zimmermann, G. S. Gazelle, and J. F. Debatin, "Radio-frequencyinduced thermoablation: monitoring with T1-weighted and proton-frequency-shift MR imaging in an interventional 0.5-T environment," Radiology, vol. 206, no. 3, pp. 803-810, Mar. 1998.

[4] L. Wang and H. Wu, "Biomedical Optics: Principles and Imaging," Hoboken, N.J.: Wiley-interscience, 2007.

[5] M. Alhamami, M. C. Kolios, and J. Tavakkoli, "Photoacoustic detection and optical spectroscopy of highintensity focused ultrasound-induced thermal lesions in biologic tissue," Medical Physics, vol. 41, no. 5, May 2014. 
[6] S. D. Kamath, S. Ray, and K. K. Mahato, "Photoacoustic spectroscopy of ovarian normal, benign, and malignant tissues: a pilot study," Journal of Biomedical Optics, vol. 16, no. 6, Jun. 2011.

[7] J. P. Gray, N. Dana, K. L. Dextraze, F. Maier, S. Emelianov, and R. R. Bouchard, "Multi-wavelength photoacoustic visualization of high intensity focused ultrasound lesions," Ultrasonic Imaging, vol. 38, no. 1, pp. 96-112, Jan. 2016.

[8] M. Xu, L. V. Wang, "Photoacoustic imaging in biomedicine," Review of Scientific Instruments, vol. 77, no. 4, 2006.

[9] X. Wu, J. L. Sanders, D. N. Stephens, Ö. Oralkan, "Photoacoustic-imaging-based temperature monitoring for high-intensity focused ultrasound therapy," in Proc. Annual International Conference of the IEEE Engineering in Medicine and Biology Society, 2016, pp. 3235-3238.

[10] U. Alqasemi, P. Kumavor, A. Aguirre, and Q. Zhu, "Recognition algorithm for assisting ovarian cancer diagnosis from coregistered ultrasound and photoacoustic images: ex vivo study," Journal of Biomedical Optics, vol. 17 , no. 12, Dec. 2012.

[11] A. Jain and D. Zongker, "Feature selection: evaluation, application, and small sample performance," IEEE transactions on Pattern Analysis and Machine Intelligence, vol. 19, no. 2, Feb 1997. 